\title{
How Sandcastles Fall
}

\author{
Thomas C. Halsey and Alex J. Levine \\ Exxon Research and Engineering, Route 22 East, Annandale, N.J. 08801
}

(March 22, 2021)

\begin{abstract}
Capillary forces significantly affect the stability of sandpiles. We analyze the stability of sandpiles with such forces, and find that the critical angle is unchanged in the limit of an infinitely large system; however, this angle is increased for finite-sized systems. The failure occurs in the bulk of the sandpile rather than at the surface. This is related to a standard result in soil mechanics. The increase in the critical angle is determined by the surface roughness of the particles, and exhibits three regimes as a function of the added-fluid volume. Our theory is in qualitative agreement with the recent experimental results of Hornbaker et al., although not with the interpretation they make of these results.
\end{abstract}

81.05.Rm, 68.45.Gd, 91.50.Jc

The continuum mechanics of most materials was established in the 19th century; however, the mechanics of granular materials is still largely mysterious [1]. The study of granular media is also motivated by the ubiquity of this form of matter in a variety of industrial contexts, as well as in geophysical ones.

While most recent attention has focussed on dry granular media, a recent experimental study by Hornbaker et $a l$. has opened the relatively unexplored subject of "humid" granular media, in which small amounts of added fluid generate, through capillarity, adhesive forces between the grains [2. Somewhat whimsically, these authors argue that their work is appropriate for the understanding of sandcastles; we actually take this point seriously, because adhesive forces and other liquid effects are extremely important in geophysical applications, of which sandcastles are an unusual example.

In this Letter, we present a theory of the stability of humid sandpiles, based upon a continuum analysis of their statics. While previous work on the statics and dynamics of dry sandpiles concentrated on the behavior of the pile's surface [3], we find that the addition of small adhesive forces between the grains causes the site of failure to move from the surface into the bulk of the sandpile, a fact well-known in soil mechanics. Even though the failure of the sandpile at the critical angle occurs at some finite depth, in the limit of infinite system size, the critical angle is actually unchanged by the adhesion. For finite systems the angle of repose is increased from the infinite-system/non-adhesive critical angle. By analyzing the cohesive effect of small amounts of wetting fluid, we find that this increase in the critical angle as a function of the added-fluid volume exhibits a range over which the dependence is linear, in agreement with the principal result of Hornbaker et al. However, we disagree with the suggestion of these authors that most of the wetting fluid will be found outside the particle contact zones.

To determine the stability of a sandpile, we must have a criterion for local failure of the sandpile. For a nonadhesive (dry) sandpile, a simple phenomenological criterion for failure is that

$$
\tau>k \sigma,
$$

where $\tau$ is the tangential stress across some plane interior to the sandpile, $\sigma$ is the normal compressive stress across that plane, and $k$ is the internal friction coefficient. For a given stress state stability requires that there be no plane for which the ratio of $\tau$ to $\sigma$ exceeds $k$. To determine the plane on which this ratio is maximized we turn to the Mohr construction [| 1 .

The Mohr circle provides a geometric construction to transform under rotations a two dimensional symmetric tensor such as the stress tensor. Suppose the two principal stresses at a point are $\sigma_{1}$ and $\sigma_{2}$. Then a circle drawn in the $\sigma-\tau$ plane through the points $\left(\sigma_{1}, 0\right)$ and $\left(\sigma_{2}, 0\right)$, symmetrically about the $\sigma$ axis, gives the normal and tangential stresses on any plane. To find these stresses for a physical system rotated by an angle $\phi$ from the principal system one looks at the points on the circle at an angle $2 \phi$ from the horizontal. These two opposite points give the normal and tangential stresses across the physical planes of the rotated system. Thus the stability criterion for a dry sandpile is that no Mohr circle may extend above the line $\tau=k \sigma$.

Consider a semi-infinite dry sandpile, whose surface is oriented at an angle $\theta$ to the horizontal. We choose an $x-z$ coordinate system, in which $z$ gives the distance from the surface of the pile ( $z>0$ down), and $x$ gives the distance parallel to the surface. Then the stress tensor $\sigma_{i j}$ satisfies the static equations

$$
\begin{aligned}
& \partial_{z} \sigma_{z z}+\partial_{x} \sigma_{x z}=\rho g \cos \theta, \\
& \partial_{z} \sigma_{z x}+\partial_{x} \sigma_{x x}=\rho g \sin \theta,
\end{aligned}
$$

where $\rho$ is the density of the sandpile. To solve these equations, we first restrict ourselves to solutions which are functions of $z$ alone-in a semi-infinite system, any $x$-dependence of the solutions would be liable to generate arbitrarily large stresses near the surface, which would cause the system to buckle. The most general $z$-dependent solution, which also satisfies the boundary condition that the surface is stress-free, is 


$$
\begin{aligned}
\sigma_{z z} & =\rho g z \cos \theta, \\
\sigma_{x z} & =\rho g z \sin \theta, \\
\sigma_{x x} & =C(z),
\end{aligned}
$$

The well-known stress indeterminacy in granular media implies that $C(z)$ is an unknown function. We can fix this function, however, by finding the form of $C(z)$ that allows us to maximize the critical angle. The angle thereby obtained will certainly be an upper bound on the true critical angle; furthermore, if the sandpile is free to adjust its undetermined stress $C(z)$ within some range, we expect that this upper bound will be identical to the critical angle. Thus, while it is impossible in general to fix $C(z)$ without some constitutive or closure relation, we do believe that it is possible to fix it at the critical angle.

Clearly the function $C(z)$ that will maximize the critical angle will have the form $C(z)=C^{\prime} z$. To apply the geometric Mohr construction, we first determine the diameter and the position of the center of the Mohr circle given by the difference and sum of eigenvalues of the stress tensor respectively. From Eqs. (46) we find that these eigenvalues are

$$
\sigma_{1,2}=z \rho g \cos \theta\left(\frac{1+c}{2} \pm \sqrt{\frac{(1+c)^{2}}{4}+\tan ^{2} \theta-c}\right),
$$

where $c=C^{\prime} /(\rho g \cos \theta)$. Note that both the radius and center position of the Mohr circle depend linearly on the depth $z$ below the surface. The maximum value of $\tau / \sigma$ as a function of $c$ is

$$
\left.\frac{\tau}{\sigma}\right|_{\max }=\sqrt{\frac{(1+c)^{2}}{4\left(c-\tan ^{2} \theta\right)}-1}
$$

Now this quantity must be $<k$, so to find the maximum critical angle, we wish to minimize the right-hand side of Eq. (8) with respect to $c$. We then find that at this minimum value of $c$,

$$
\left.\frac{\tau}{\sigma}\right|_{\max }=\tan \theta
$$

so that the critical angle is $\theta_{c}=\tan ^{-1} k$, which is a classic result [5]. This simple model, by itself, does not indicate at which depth failure initiates-presumably a dynamical model would resolve this ambiguity [6].

Now consider a sandpile in which a normal adhesive stress $s_{A}$ is exerted across every plane, in addition to whatever other stresses may exist due to the body forces. This stress introduces a normal force between pairs of contiguous particles which allows the sandpile to support a finite shear stress, even in the limit of zero applied compressive stress. The maximum supported shear stress, in this case, is $k s_{A}$ and we therefore replace the dry sand failure criterion, Eq. (1), by

$$
\tau>k\left(\sigma+s_{A}\right) \text {. }
$$

Performing a calculation similar to the one above, we find that the failure criterion, which is now an explicit function of depth, is

$$
k=\tan \theta\left(1+\frac{s_{A}}{\rho g z \cos \theta}\right)^{-1} .
$$

Note that since the sandpile will only be in a state of incipient failure below some fixed depth, the stress tensor retains some indeterminacy above that depth. Our Mohr analysis, now local, applies only at the incipent failure depth and does not determine the global stress state of the sand pile.

The criterion thus derived, Eq. (11), is most stringent as $z \rightarrow \infty$, in which case the dry sandpile result $\theta_{c}=$ $\tan ^{-1} k$ is recovered. On the other hand, for a sandpile of fixed depth $D$, the failure must occur at most at depth $D$. Thus the critical angle will be the solution of the equation

$$
k=\tan \theta_{c}(D)\left(1+\frac{s_{A}}{\rho g D \cos \theta_{c}(D)}\right)^{-1},
$$

giving an critical angle $\theta_{c}(D)$ that decreases monotonically with $D$. Thus finite humid sandpiles have a depthdependent critical angle, unlike dry sandpiles, which is a well-known result in soil mechanics [5]. In addition, we see that humid (i.e. cohesive) sandpiles fail at depth, whereas from statics alone we were unable to determine the failure depth of dry sandpiles. In the case of small adhesion stress $s_{A} / \rho g D \ll 1$, we can write

$$
\tan \theta_{c} \approx k+\frac{k s_{A}}{\rho g D} \sec \left[\tan ^{-1}(k)\right] .
$$

If, as we are assuming, the adhesion arises from capillary forces, we must still connect the adhesive stress to the amount of fluid present. We suppose that the sand is composed of macroscopically spherical grains (radius $R$ ) whose surface roughness may be characterized as follows: the spatial correlation of fluctuations in local surface height saturates at height $l_{R}$ at a lateral distance $d$ that is much smaller than the particle radius, $d \ll R$. Since the particles are macroscopically spherical, we require that $l_{R} \ll R$ (see Fig. 11).

We can characterize the surface roughness of two particles in contact by considering the function $\delta(x)$ which gives the average distance between the two particles a lateral distance $x$ from an asperity at which the two particles are in contact. We write $\delta(x)$ in the form [7]

$$
\delta(x)=l_{R} f(x / d)
$$

where $f(w)$ is a scaling function with the limits

$$
f(w) \sim \begin{cases}w^{\chi} & w \rightarrow 0 \\ 1 & w \rightarrow \infty\end{cases}
$$

The roughness exponent, $\chi$, of the surface satisfies $0<$ $\chi \leq 1$. 


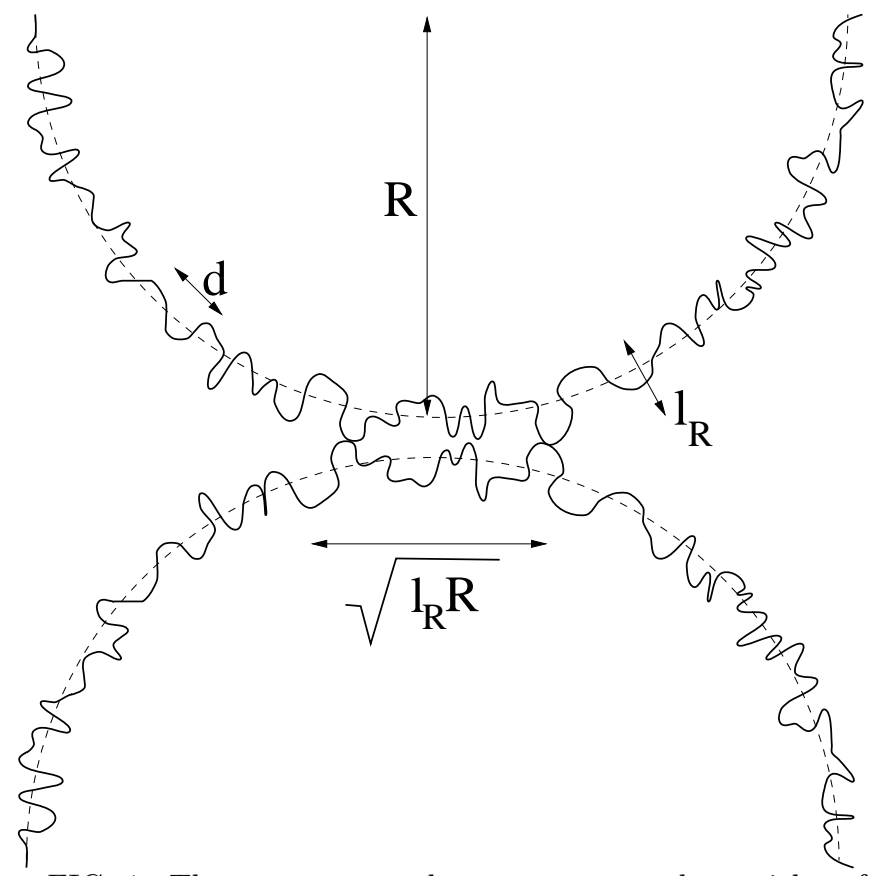

FIG. 1. The contact zone between two rough particles of radius $R$. The scale of height deviations from the mean is $l_{R}$ and the height fluctuations are correlated over a distance $d$. The lateral size of the contact zone in which the macroscopic curvature of the particles is not apparent is $\sim \sqrt{l_{R} R}$.

Note that this form can only be valid for $x<\sqrt{l_{R} R}$. For larger values of $x$, the macroscopic curvature of the particles will determine the local distance between them (see Fig. 1).

For such particles, there are three regimes for the capillary force exerted by a wetting fluid as a function of $V$, the total amount of fluid present per particle contact.

Asperity Regime- For the smallest values of $V$, the capillary force is dominated by the accumulation of fluid around a single or a small number of asperities at which two neighboring particles are in contact. This will hold until the lateral extent of the fluid-filled region exceeds $d$, determining a maximum contact fluid volume for this regime, $V_{1} \sim l_{R} d^{2}$.

We can write the adhesion force $f_{A}$ as

$$
f_{A}=\frac{\Gamma A}{r},
$$

where $\Gamma$ is the surface tension of the fluid, $r$ is the radius of curvature of the meniscus of the fluid layer connecting the two grains near the asperity and $A \sim V / \delta$ is the area of the contact patch. $-\Gamma / r$ is the pressure reduction due to the capillary meniscus. Because $r$ will be approximately the distance between the particles at the meniscus, we find

$$
P \sim-\frac{\Gamma}{l_{R}}\left(\frac{V}{V_{1}}\right)^{-\frac{\chi}{2+\chi}},
$$

and the adhesion force is

$$
f_{A} \sim \frac{\Gamma V_{1}}{l_{R}^{2}}\left(\frac{V}{V_{1}}\right)^{\frac{2-\chi}{2+\chi}}, \text { for } V<V_{1},
$$

where $V_{1}=l_{R} d^{2}$.

For a rough surface where $\chi=1$, Eq. (18) shows that the force depends on the cube root of the fluid volume. This is identical to the dependence of the cohesive force between a cone and plate on the volume of the liquid bridge connecting them [2]. It is to be expected that the cone-and-plate model will reproduce the cohesive force near a single asperity.

Roughness Regime- For larger values of $V$, the fluid will occupy a statistically rough region, which is still small enough that the macroscopic curvature of the particles plays no role-however, the fluid occupies more than the area around a single asperity. This regime occurs for $V_{1}<V<V_{2}$, where $V_{2}=l_{R}^{2} R$. The pressure is

$$
P \sim-\frac{\Gamma}{l_{R}},
$$

and the force will be

$$
f_{A} \sim \frac{\Gamma V}{l_{R}^{2}}, \text { for } V_{1}<V<V_{2},
$$

In this roughness regime, the cohesive force is linear in the volume of the added fluid, reproducing the linear dependence found by Hornbaker et al.

Spherical Regime- When the lateral extent of the fluid contact exceeds $d$, then the wetting region will be determined by the macroscopic curvature of the particles, and the surface roughness will no longer play a significant role. In this case the pressure is given by [8]

$$
P=-\frac{\Gamma}{\sqrt{V / 2 \pi R}},
$$

and the force by

$$
f_{A}=2 \pi \Gamma R, \text { for } V>V_{2},
$$

which is independent of the volume of the liquid bridge joining the two grains. Thus the linear increase of the cohesive force with fluid volume saturates for volumes $V>V_{2}=l_{R}^{2} R$ (see Fig. 2).

If the fluid wets the surface of the particles, then in addition to the fluid in the contact region, there will also be a layer of fluid of thickness $t$ on the surface of the particles. Typically this film will be no thicker than a few monolayers; hence there are a complicated set of forces between this film and the surface. To simplify, we consider only the Van der Waals forces, which for a thickness $t$ generate a "disjoining pressure" $P_{d}$ given by [9]

$$
P_{d}=\frac{2 H}{t^{3}},
$$

where $H$ is the Hamaker constant $(H<0$ for a wetting fluid). To determine the thickness of the wetting region, 


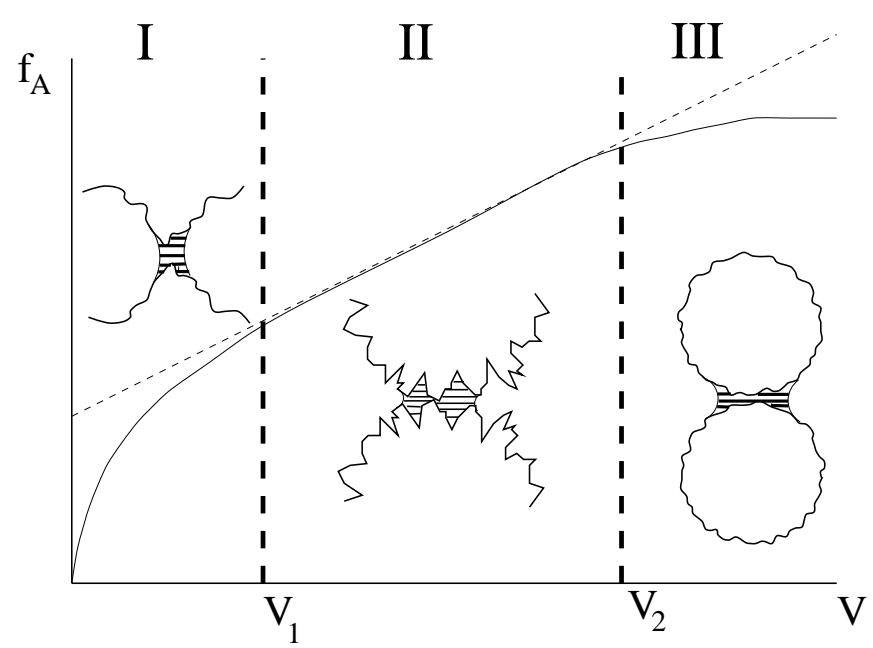

FIG. 2. The behavior of the adhesive force between two rough, "spherical" particles. The three regimes of the force vs. volume of the wetting layer are I - Asperity Regime, II - Roughness Regime, and III - Spherical Regime. The insets show the extent of the wetting region typical of each regime.

we must set this disjoining pressure equal to the pressure inside the contact regions. If the radius of curvature of the contact meniscus is $r$, then

$$
t=\left(-\frac{2 H r}{\Gamma}\right)^{\frac{1}{3}} .
$$

In the asperity regime, $t$ will increase with the meniscus radius of curvature. In the roughness regime, however, $r$ saturates at a value $\sim l_{R}$, and $t$ will be constant. In this regime any added fluid enters the contact region. Finally, in the spherical regime, $t$ will again increase.

Now consider a system with a volume $V_{l}$ of liquid per particle. If we suppose that the spheres are close-packed, then each sphere has 12 neighbors, so there is an average of 6 contacts per sphere, each with a fluid volume of $V=V_{l} / 6$. The average number of contacts per unit area will be $\left(3 \phi_{V} / \pi R^{2}\right)$, where $\phi_{V}$ is the volume fraction of the particles. Thus for a close-packed lattice, for which $\phi_{V}=\sqrt{2} \pi / 6$, the adhesive stress will be approximately

$$
s_{A} \approx \frac{f_{A}}{\sqrt{2} R^{2}},
$$

and we can now substitute into Eq. (13) to obtain the dependence of the critical angle upon fluid volume. For a sandpile of fixed depth $D$, this is linear in $V$,

$$
\tan \theta_{c} \approx k+\frac{k \Gamma V}{\sqrt{2} l_{R}^{2} R^{2} \rho g D} \sec \left[\tan ^{-1}(k)\right],
$$

up to a saturation result determined by (see Fig. 2)

$$
\tan \theta_{c} \approx k+\frac{\sqrt{2} \pi k \Gamma}{R \rho g D} \sec \left[\tan ^{-1}(k)\right] .
$$

Now let us consider the experiment of Hornbaker et al. They measured the critical angle for a medium composed of radius $4 \times 10^{-2} \mathrm{~cm}$ polystyrene spheres with small amounts of added oil using the draining crater method [2]. They found a linear increase in the critical angle measured as a function of the volume of added oil. They claimed that the failure of their systems was at the surface, and concluded that they could account for their results by assuming that $99.9 \%$ of the fluid was outside of the contact zones between the particles. Their particles had a surface roughness on the order of $1 \mu \mathrm{m}$.

We find that the increase in the critical angle is linear with the fluid volume in the roughness regime, up to the saturation result Eq. 27). We expect the Hamaker constant for a wetting fluid to be negative, and of the order of magnitude of $H \sim 10^{-20} \mathrm{erg}$, so no more than a few monolayers of fluid should be present on the particle surfaces. Furthermore, in the linear regime, all fluid added to the system will enter the particle contacts. Thus we disagree with the claim that the overwhelming majority of the fluid in this case will be outside the contact zones. Finally, we disagree with the interpretation of their experiment, according to which surface failure is the most relevant failure mode- the failure plane in cohesive materials should be at depth.

We are grateful to P. Schiffer for providing us with copies of Ref. [2] before publication and to D. Ertaş for many useful discussions.

[1] H.M. Jaeger, S.R. Nagel, and R.P. Behringer, Rev. Mod. Phys. 68, 1259 (1996).

[2] D.J. Hornbaker, R. Albert, I. Albert, A.-L. Barabási, and P. Schiffer, Nature 387, 765 (1997), and unpublished.

[3] J.-P. Bouchaud, M. E. Cates, J. R. Prakash, and S. F. Edwards, J. Phys. I, 4, 1383 (1994).

[4] A.C. Ugaral and S.K. Fenster, Advanced Strength and Applied Elasticity (Prentice-Hall, Englewood Cliffs, NJ, 1987) Chapter 1.

[5] R.M. Nedderman, Statics and Kinematics of Granular Materials (Cambridge University Press, New York, 1992) Chapter 3.

[6] H.M. Jaeger and S.R. Nagel, Science 255, 1523 (1992).

[7] A.-L. Barabási and H.E. Stanley, Fractal Concepts in Surface Growth (Cambridge University Press, New York, 1995) Chapter 2.

[8] F.P. Bowden and D. Tabor, The Friction and Lubrication of Solids, (Oxford University Press, 1986), Chapter 15.

[9] S. A. Safran, Statistical Thermodynamics of Surfaces, Interfaces, and Membranes (Addison - Wesley Publishing Co., New York, 1994) Chapter 5. 